\title{
Publisher's Note: Demonstrating universal scaling for dynamics of Yukawa one-component plasmas after an interaction quench [Phys. Rev. E 93, 023201 (2016)]
}

T. K. Langin, T. Strickler, N. Maksimovic, P. McQuillen, T. Pohl, D. Vrinceanu, and T. C. Killian (Received 14 April 2016; published 25 April 2016)

DOI: 10.1103/PhysRevE.93.049905

This paper was published online on 3 February 2016 with typographical errors on page 1 and 4. On page 1, left-hand column, the first sentence of the second paragraph should read as "One important feature of the Yukawa model is that, in suitably normalized units, dynamics depend solely on the screening parameter, $\kappa=a / \lambda_{D} \ldots$.." On page 4 , left-hand column, the fourth sentence of the second paragraph should read as "For the work discussed in this paper, the effect is relatively minor, as $t_{\mathrm{DIH}}$ is shorter than $\tau_{\exp }=\sqrt{m_{i} \sigma_{0}^{2} /\left(k_{B} T_{e}\right)}$, the time scale for expansion." The paper has been corrected as of 14 April 2016 . The text is incorrect in the printed version of the journal. 